\title{
Water Security Risk Assessment of the Yellow River Basin Based on Constrained-Random Weight and Cloud Model
}

\author{
Song Chenye, Zhang Ren*, Zhou Aixia, Hong Mei \\ College of Meteorology and Oceanography, PLA University of Science and Technology, Nanjing 211101, China \\ *E-mail: songchenye@126.com; zren63@126.com \\ Received 29 October 2013 \\ Accepted 12 December 2013
}

\begin{abstract}
Present risk assessment methods so far were majorly based on the primary knowledge and were incapable of combining uncertainty into risk assessment outputs. In order to solve this problem, a new methodology for risk assessment using constrained-random weight method and cloud model is introduced here to perform the risk assessment of water security in the Yellow River basin. The effect of knowledge deficiency on risk assessment can be illustrated on the instability of risk series by the constrained-random weight method; the result of assessment can incorporate the randomness and fuzziness of risks and corresponding assessments, and transition of quantified risk series into qualitative risk level can be possible as the presence of the cloud model. Experiment about the water security risk assessment of the Yellow River baisin proved the validity and applicability of this method.
\end{abstract}

Keywords: Water Security;Cloud Model;Constrained-Random Weight;Risk Assessment

\section{基于随机赋权法和云模型的黄河流域水安全风险评估 宋晨烨 张㓞 周爱霞 洪梅 \\ 解放军理工大学气象海洋学院, 江苏 南京 211101}

\begin{abstract}
摘要：针对现有风险评估方法大多着眼于已有知识背景下对风险大小的评估, 难以将不确定性融入评估结 果的问题, 引入约束随机赋权和云模型相结合的方法途径, 对黄河流域的水安全风险进行了评估。约束随 机赋权法的引入可将知识不完备性对风险评估的影响体现于风险序列的不稳定性上, 云模型方法的引入使 评估结果可包含风险及风险评估的随机性和模糊性信息, 并将定量的风险序列与定性的风险等级区划合理 转换。以黄河流域为例, 建立了水安全风险评估的指标体系和风险评估模型, 并进行了仿真试验, 验证了 方法的有效性和适用性。
\end{abstract}

关键词: 水安全, 云模型, 随机赋权, 风险评估

\section{1. 引言}

风险评估工作的目的是为决策者提供决策建议。 目前, “风险” 尚未有明确统一的定义, 纵观现有观点, 离不开风险是危险 (不安全) 程度 “大小” 及其发生 可能性的 “不确定性” 结合的分析途径 ${ }^{[1]}$ 。

目前的风险评估研究主要是通过建立指标体系, 对指标赋权再通过相应评估方法对风险大小进行评 估。目前指标赋权主要有主观赋权法和客观赋权法, 以及主客观综合的赋权方法。主观赋权法由于主观随 意性过强, 其结果受打分专家的主观性影响较大使其 风险评估的科学性不稳定; 而客观赋权法则存在着评 估结果解释性和适用性差的缺点, 所得的结果难以给 出明确的解释 ${ }^{[2-3]}$ 。无论何种赋权方法, 都旨在寻求一 种给予指标体系一个合理的权重分配。但由于在风险 识别阶段, 风险因子的作用机制常不明确, 只能得到
其中的部分致险因子相对重要程度。鉴于客观现实条 件下的知识不完备性, 以及风险本身模糊性和随机性, 用精确的权重来反映各指标的重要性, 进而对风险进 行分析和评估, 将会忽略这种不确定性, 反而不能合 理反映风险因子对风险的作用 ${ }^{[4]}$ 。随机赋权法通过重 复试验产生权重序列, 将现有知识信息充分利用, 同 时将知识的不完备性和风险的随机性体现于风险序列 中。目前随机赋权法已成为一种常用的综合评价方法。 另一方面, 将风险量化成一个确定的数值, 一定程度 上有利于人们对风险大小进行理解, 但单一的数值并 不能代表风险的全部特征, 在实际应用中, 需要寻找 一个更能全面体现风险特征的评估方法, 可以在给出 定量结果的同时也给出易于直观把握的定性特征。常 用的定性定量转换方法中, 如层次分析法、专家群体 打分法、控制论和定性分析等, 不能同时兼顾随机性 和模糊性, 李德毅院士提出的云理论可将语言值表示

Published by Atlantis Press

Copyright: the authors 
的定性概念和其定量表示之间的不确定性联系起来, 把风险在不确定性问题上的模糊性和随机性集成到一 起，构成定性概念和定量数值之间映射，目前，云理 论已被广泛应用于复杂系统的综合评估之中。近十年 来, “水安全” 概念在政治领域和学术领域都得到广泛 的重视 ${ }^{[5]}$, 由于水安全是一个涉及水文学、环境科学、 经济学、哲学等多学科领域的复杂概念, 同时涉及政 治和政策决策，不同专业背景的研究者对其研究角度 不同，目前还没有统一的定义，其内涵主要包括人类 生存与发展不存在水资源问题的危险和威胁的状态, 外延则与国家安全相关, 包括满足人类需求、粮食供 应、生态系统安全、水资源供需、水灾害风险管理、 社会安全与稳定等 ${ }^{[6]}$, 涵盖了资源、环境、生态、社 会、政治、经济等多方面。本文所指的水安全以人类 社会的稳定和发展对水的需求为水安全问题的主体, 水资源为客体, 以黄河流域的水安全为例, 引入随机 赋权法, 将现有知识信息充分利用, 同时也将知识的 不完备性和风险的随机性融入评估结果之中; 云模型 方法主要用于定性概念和定量数值的转化, 在风险序 列分析及获取评估对象风险不同特征等方面, 表现出 较好的客观性和适用性。

\section{2. 水安全风险评估指标体系}

黄河是我国第二大河，整体位于我国中北部，自 西向东分为三个地形阶梯, 其中最高级海拔较高, 属 于高寒地区, 土地覆盖以牧草地为主, 水质受水污染 影响较小; 第二阶梯地势落差超过 $1000 \mathrm{~km}$, 水文气象 现象复杂, 包括几个较大的地貌单元, 其中水土流失 较为严重的黄土高原是黄河中游洪水和泥沙主要来源 区; 第三阶梯为黄河冲击大平原, 地势平坦, 大量泥 沙在此淤积, 排水条件差, 旱涝灾情严重。黄河流域 大多数地区属于干旱半干旱气候区, 蒸发强度高, 流 域内水资源紧缺, 不同区间径流量悬殊, 兰州站以上 和三门峡至花园口区间水资源量丰富, 兰州站至花园 口之间地区水资源贫乏, 是干旱事件的多发区。整体 而言, 黄河流域的水资源量、气候特点、地理环境以 及社会结构、经济发展、人口分布都存在着严重的分 布不均现象, 例如, 龙门以下的地区占有黄河流域人 口数量的 $70 \%$, 然而该区域面积只占全流域 32\%。水 资源短缺、生态环境脆弱、水土流失严重以及社会发 展结构有待调整是黄河流域水资源管理工作的重点。

对风险评估工作而言, 无论采用何种评估方法, 首先要建立一个合理的风险评估指标体系 ${ }^{[7]}$ 。水安全 评估系统是一个由众多子系统构成的复杂系统, 具有 层次性, 指标体系设置须考虑区域自身特点、水资源 系统特性、可操作性以及实际可获得的数据 ${ }^{[8]}$ 。按照 构建指标的科学性、完备性、动态性与静态性结合、 定性与定量结合及可比性、可操作性等原则 ${ }^{[9]}$, 以水 安全风险作为指标体系的目标层, 将风险的致灾因子 危险性, 承灾体脆弱性, 风险防范能力三个方面作为 指标体系的准则层 ${ }^{[10]}$ 。致灾因子的危险性表示导致风 险的直接因素; 承灾体脆弱性表示在致灾因子作用下， 承灾体受危害影响的程度; 风险防范能力是指承灾体 对致险因子的抵御和防范, 以减轻灾害后果的能力。
参考前人的工作 ${ }^{[11-14]}$ ，从水资源利用过程中 “自然” 和 “社会” 两个角度出发, 针对黄河流域水资源短缺、 中下游水土流失严重的特点, 对危险性构建包括水资 源本身的丰沛程度、水资源可利用程度、水资源受污 染程度三个一级指标的指标体系; 对承灾体脆弱性从 暴露性和敏感性两个一级指标, 从经济, 人口, 农业 进行分析, 构建相应的二级指标; 风险防范能力, 将 水利工程调节能力、节水能力、废水处理能力作为一 级指标, 最终得到的指标体系见表 1 。

\section{表1 黄河流域水安全风险评估指标体系}

Table 1 Risk assessment index system of the Huang River

\begin{tabular}{|c|c|c|c|c|}
\hline \multicolumn{5}{|c|}{ basin water security } \\
\hline 目标层 & 准则层 & 一级指标 & 二级指标 & 序号 \\
\hline \multirow{17}{*}{$\begin{array}{l}\text { 水资源 } \\
\text { 安全 }\end{array}$} & \multirow{6}{*}{$\begin{array}{c}\text { 致灾因 } \\
\text { 子 } \\
\text { 危险性 }\end{array}$} & \multirow{2}{*}{$\begin{array}{c}\text { 水资源丰 } \\
\text { 沛度 }\end{array}$} & 地下水资源量 & 1 \\
\hline & & & 地表水资源量 & 2 \\
\hline & & $\begin{array}{l}\text { 水资源可 } \\
\text { 利用程度 }\end{array}$ & 城市面积比 & 3 \\
\hline & & \multirow{3}{*}{$\begin{array}{c}\text { 水污染程 } \\
\text { 度 }\end{array}$} & $\begin{array}{l}\text { 生活污水 } \\
\text { 排放量 }\end{array}$ & 4 \\
\hline & & & $\begin{array}{l}\text { 工业污水 } \\
\text { 排放量 }\end{array}$ & 5 \\
\hline & & & 农药施用量 & 6 \\
\hline & \multirow{6}{*}{$\begin{array}{l}\text { 承灾体 } \\
\text { 脆弱性 }\end{array}$} & \multirow{4}{*}{ 暴露性 } & 人口数量 & 7 \\
\hline & & & 人口增长率 & 8 \\
\hline & & & 评估单元 GDP & 9 \\
\hline & & & 耕地面积 & 10 \\
\hline & & \multirow{2}{*}{ 敏感性 } & 农业用水量 & 11 \\
\hline & & & 人均用水量 & 12 \\
\hline & \multirow{5}{*}{$\begin{array}{c}\text { 风险防 } \\
\text { 范 } \\
\text { 能力 }\end{array}$} & \multirow{2}{*}{$\begin{array}{l}\text { 水利工程 } \\
\text { 调节能力 }\end{array}$} & $\begin{array}{c}\text { 大中型水库 } \\
\text { 个数 }\end{array}$ & 13 \\
\hline & & & $\begin{array}{c}\text { 大中型水库 } \\
\text { 储水量 }\end{array}$ & 14 \\
\hline & & \multirow[b]{2}{*}{ 节水能力 } & 水重复利用率 & 15 \\
\hline & & & $\begin{array}{l}\text { 节水灌溉 } \\
\text { 面积比 }\end{array}$ & 16 \\
\hline & & 废水处理 & $\begin{array}{c}\text { 污水处理厂污 } \\
\text { 水处理比 }\end{array}$ & 17 \\
\hline
\end{tabular}

\section{3. 约束随机赋权法}

权重是指标重要性的量化表示, 可根据目标的重 要性赋予不同的值, 且所有指标权重之和应等于 $1^{[15]}$ 。 但由于知识不完备性和风险本身的随机性及其他各 15 不确定性, 各个指标之间具体的相互关系无法得到明 确的结论, 此时若对指标赋予精确的权值不仅会使赋 权结果失真, 还会导致评估结果可参考性降低 ${ }^{[16]}$ 。

约束随机赋权将风险的知识不完备性和指标之间 关系的模糊性通过随机对指标赋权的方式体现; 对指 标随机赋权重复多次可以体现风险因子作用的随机 性; 根据风险已知的并且确定的知识, 通过对指标体 系分析并以约束条件的形式对随机赋权进行约束。以 在约束条件下的随机多次赋权, 模拟本身具有模糊性

Published by Atlantis Press

Copyright: the authors 
宋晨烨，张㓞，周爱霞，等

各个风险因子的随机作用产生风险的过程, 得到的风 险序列则可包含评估对象风险的总体特征信息。

\section{1 指标分析和约束条件确定}

考虑指标间的部分可知性，在专家建议的基础上， 对已知部分作有弹性约束, 对相互作用以及重要性程 度无法明确对比的部分指标随机生成。具体方法如下:

若 $\left(\omega_{1}, \omega_{2}, \cdots, \omega_{p}\right)$ 是指标体系的 $p$ 个评估指标的 权重值, 根据指标权重的定义, 所有指标权值在 0 和 1 之间, 且所有指标权重之和为 1 , 由此产生约束条件:

约束 $1: \sum_{j=1}^{p} \omega_{j}=1$, 且 $\omega_{j} \in[0,1]$ 。

其他的约束条件, 可以结合对风险因子的分析和 专家的建议, 根据对指标体系最大程度的掌握得到。 在确定约束条件的过程中, 约束条件个数没有硬性范 围, 但给出约束条件的前提是可以较为明确地判断指 标之间重要性程度的关系, 对于无法明确判断的, 不 必给出约束。

本研究以 $\left(\omega_{1}, \omega_{2}, \cdots, \omega_{17}\right)$ 代表水安全指标体系中 17 个指标的权重。除基本约束条件约束 1 外, 根据对 水安全风险因子分析, 结合专家建议, 可以得到针对 黄河流域水安全风险指标体系的指标权重约束条件:

约束 2: $\omega_{6}<\left(\omega_{4}, \omega_{5}\right)$; 水污染的主要来源是人类 工业的发展和人口的增多导致的生活污水排放增加, 而农业生产对水资源和水循环的影响主要是由于对水 资源的消耗以及下垫面植被覆盖的改变导致的水循环 改变, 因此在 “水污染程度” 的三个指标中, “农药施 用量” 的权重比 “生活污水排放量” 和 “工业污水排 放量”小

约束 3: $\omega_{10}<\left(\omega_{7}, \omega_{8}, \omega_{9}, \omega_{11}, \omega_{12}\right)$; 虽然农业用水 是水资源开发利用中耗水最大的方向, 但考虑农业生 产的经济产值较工业生产较低, 其对水安全风险的暴 露性应较小, 因此将耕地面积在所有承灾体脆弱性的 指标中的权重最小。

约束 4: $\max \left(\omega_{13} \sim \omega_{17}\right)<\min \left(\omega_{1} \sim \omega_{12}\right)$; 黄河流 域对水安全问题的防范能力仍然较弱, 风险的大小更
多是受自然资源和自然环境本身的危险性以及人类社 会系统脆弱性的影响, 因此风险防范能力的指标权重 应小于致险因子危险性和承灾体脆弱性的指标权重。

\section{2 权重矩阵的生成}

约束随机权重法不明确给出所有指标的具体权 重, 对一个有 $p$ 个指标组成的指标体系, 设生成的指 标序列组数为 $m$ (本研究中取为 100 ), 则在约束条件 下随机生成相当规模的矩阵 $\left(\omega_{i j}\right)_{m \times p}, \omega_{i j}$ 表示第 $j$ 个 指标的第 $i$ 次随机生成权重值。以表 1 中所列的 17 个 指标组成的指标体系为例, 具体做法如下:

(1) 由 Matlab 中 random 函数生成 $m=100$ 个容 量为 $p=17$ 的一维随机数组, 得到规模为 $100 \times 17$ 的随 机矩阵;

(2) 依上一步得到的约束条件调整每一行数组元 素的排序, 使矩阵每一行数组都满足 2.1 所列的约束 条件;

(3) 将所有行的行数组归一化, 得到满足约束条 件的随机矩阵 $\left(\omega_{i j}\right)_{100 \times 17}$, 即指标权重矩阵 (表 2)。

表 2 中的每一组权值序列仅代表一种可能的权值 分配。由于权值分配的不确定性是知识不完备和致险 因子本身的不确定性造成的, 而知识不完备性同样也 是风险的一部分, 因此在约束条件下产生的各种权值 序列在风险的产生机制上是等可能的。

须说明的是, 上述的操作方法得到的权重序列并 不是按先确定约束条件, 再随机生成指标值的顺序得 到, 而是用约束条件对已生成的随机数组调整, 进而 给指标权值赋值。同时, 由于在计算机上产生的随机 数都是按照一定的计算方法产生的, 不可能是真正的 随机数 ${ }^{[1]}$, 事实上得到的只是 “伪随机数”。然而, 对 指标值随机产生大量权重序列的意义在于在已知条件 下最大限度模拟风险产生的不同可能, 这样的得到的 伪随机数列可以很大程度上实现对风险的模拟, 因此 上述的操作方法仍是可用的。

表 2随机赋权法生成的部分权重序列

Table 2 Risk series generated by constrained-random weight

\begin{tabular}{cccccc}
\hline \multicolumn{7}{c}{ 第一组权值序列 $\omega_{1 j}$} \\
\hline 0.035454 & 0.089632 & 0.110402 & 0.065976 & 0.042139 & 0.034933 \\
0.148333 & 0.063914 & 0.026279 & 0.021192 & 0.174896 & 0.135357 \\
0.01558 & 0.003082 & 0.016746 & 0.010018 & 0.006068 & \\
\hline \multicolumn{7}{c}{ 第二组权值序列 $\omega_{2 j}$} \\
0.092578 & 0.087565 & 0.088072 & 0.071285 & 0.07504 & 0.066425 \\
0.05521 & 0.101312 & 0.050307 & 0.048926 & 0.064301 & 0.045173 \\
0.018263 & 0.018857 & 0.030383 & 0.04173 & 0.044573 & \\
\hline \multicolumn{7}{c}{$\ldots . .}$.
\end{tabular}

\section{3 风险序列的生成}

$a_{j}(j=1 \cdots p)$ 表示指标体系的 $p$ 个指标, 共有 $n$ 个 评估对象。指标的量化值结果为 $\left(\mu_{j k}\right)_{p \times n}\left(j=1 \cdots p ; k=1 \cdots n, \mu_{j k}\right.$ 指第 $k$ 个评估对象的
第 $j$ 个指标量化结果。根据随机生成的指标权重 $\left(\omega_{i j}\right)_{m \times p}$, 可得到风险矩阵:

$$
\left(R_{i k}\right)_{m \times n}=\left(\omega_{i j}\right)_{m \times p} \times\left(\mu_{j k}\right)_{p \times n},
$$

Published by Atlantis Press

Copyright: the authors 
$R_{i k}$ 表示根据第 $\mathrm{i}$ 组指标权重值对第 $k$ 个评估对象的评 估结果,即 $\left(R_{i k}\right)_{m \times n}$ 的每一列代表对应的评估对象的风 险序列,每一行则对应一次指标赋权情况下所有评估 对象的风险。

本研究中, 根据指标量化结果存放于矩阵 $\left(\mu_{j k}\right)_{p \times n}, p=17, n=8$ (8 个评价对象/省)。其中人口 数据来自各省第六次全国人口普查数据公报, 水资源 量, 水污染水利工程相关数据来自《中国环境统计年 鉴 2011》。由公式:

$$
\left(R_{i k}\right)_{m \times n}=\left(\omega_{i j}\right)_{m \times p} \times\left(\mu_{j k}\right)_{p \times n}, m=100, n=8, p=17
$$

得到。表 3 列出了在 2.2 中得到的 100 组权值序列对 应的 100 次指标融合得到的部分风险值, 每一个评价 对象的 100 个风险值组成该评价对象的风险序列。

每一次赋权得到的风险值代表一种可能的致险机 制产生的风险值; 不同的赋权导致的风险序列的波动 既包括了自然条件致险因子本身的不确定性, 也包含 了知识不完备产生的风险。也可以认为, 风险序列本 身就是现有知识水平下的评价对象量化以后的风险。

表 3 黄河流域水安全风险序列（部分）

Table 3 Water security risk series of the Yellow River basin

\begin{tabular}{|c|c|c|c|c|c|c|c|c|}
\hline 赋权次数 & 山西 & 内蒙古 & 山东 & 河南 & 陕西 & 甘肃 & 青海 & 宁夏 \\
\hline 1 & 0.591319 & 0.60923 & 0.650574 & 0.597163 & 0.497486 & 0.584994 & 0.118856 & 0.445937 \\
\hline 2 & 0.604665 & 0.530121 & 0.605908 & 0.542874 & 0.479991 & 0.510023 & 0.13183 & 0.486615 \\
\hline 3 & 0.648038 & 0.491033 & 0.633445 & 0.551778 & 0.488425 & 0.498057 & 0.064303 & 0.506169 \\
\hline 4 & 0.636975 & 0.56264 & 0.670558 & 0.594341 & 0.49139 & 0.530755 & 0.068905 & 0.481557 \\
\hline 5 & 0.598728 & 0.53621 & 0.603228 & 0.508707 & 0.429022 & 0.540665 & 0.101174 & 0.563585 \\
\hline 6 & 0.552852 & 0.522702 & 0.55233 & 0.477412 & 0.40931 & 0.565649 & 0.124374 & 0.538443 \\
\hline ...... & ...... & ...... & ...... & ...... & ...... & ...... & ...... & ...... \\
\hline 100 & 0.616333 & 0.544584 & 0.646446 & 0.576116 & 0.490108 & 0.519891 & 0.086582 & 0.476727 \\
\hline
\end{tabular}

\section{4. 云模型风险区划建模}

风险序列可看作是量化的风险（包括危险程度的 大小和不确定性), 但单纯的数字序列通常无法体现风 险特征, 也无法对辅助决策起到有效的帮助。因此将 风险序列与人类在衡量事物好坏时所习惯使用的语言 值(如“好”，“坏”)对应起来，是风险区划必须的工作。

云 “远看有形，近看无边” 的特征同人类对事物 的理解和评价思维方式有相似处。对于某一个语言值, 人们通常只能大致判断哪些属于“好”, 哪些属于 “坏”, 但不会给出精确的边界。因此, 云也可以看作是人类 的语言值和自然的数字值之间互相对应的桥梁。

云模型由李德毅院士提出, 是用语言值表示的定 性概念与其定量表示之间的不确定性转换模型 ${ }^{[18]}$ 。设 $U$ 是用精确数值表示的定量论域, $C$ 是 $U$ 上的定性概 念, 对 $\forall x \in U$ 存在一个有稳定倾向的随机数 $y(x) \in[0,1]$, 表示 $x$ 对 $C$ 的确定度, 则每一个 $x$ 称为 一个云滴, $x$ 在论域 $U$ 上的分布称为云, 用期望 $E x$, 熵 $\mathrm{En}$ 和超熵 $\mathrm{He}$ 三个数字特征来整体表征一个概念 [19-23]。期望值代表了云的整体属性，熵和超熵则是对 云的形状的描述：熵是对云代表的定性概念确定程度 的度量, 描述了云的离散程度, 在最后得到的风险云 图中显示为风险云的覆盖范围; 超熵则是熵的不确定 程度, 也就是熵的熵, 可认为是对云的厚度的描述。 云模型方法适用于对某一语言概念在定性和定量上的 转化, 可用于对已生成的风险序列做进一步分析, 将 风险序列的量化数组对应成一朵语言上的评估云, 最
终将评估对象的风险序列和评估结果 (评语) 对应起 来, 并可进一步做风险的区划。

利用云模型的风险评价方法，首先将水安全风险 分为由好至差的奇数个语言等级, 称为评语集。每个 风险等级对应一个评估云。所有的评估云置于同一个 坐标构成评估的云标尺。将某一评估对象的风险云与 云标尺对比即可得到的评估结果。最终所获得的风险 云, 其期望值 $E x$ 代表了评估对象所具有的风险的整体 水平, 熵 $E n$ 代表了风险的离散程度, 可以认为是评估 对象具有的风险对于某一评语等级的确定程度, 风险 云离散程度越高, 对应于某一评语等级的确定程度越 低。超熵 $\mathrm{He}$ 是风险云的厚度, 代表评估对象所属的评 语等级在语言上的模糊性。

\section{1 评语集的生成}

云标尺的获得首先要得到各评估云的三个特征 值, 再将特征值用正向云发生器云化而得。本研究中, 将水安全风险分为九个语言等级, 对应的语言值为 $[$ 极 好, 很好, 好, 较好, 中等, 较差, 差, 很差, 极差]。 其中 [极好, 好, 中等, 差, 极差]五个等级的评语集 由变量云化过程和云发生器得到对应的评估; 对 [很 好, 较好, 较差, 很差]四个评语集, 则利用浮动云得 到。具体做法如下:

\subsection{1 定量变量云化得到评语集}

根据云的定义, 对某一过程表示定性概念的定量 变量进行云描述, 包括获取云的数字特征和形状, 这 一过程被称为变量云化过程 ${ }^{[24]}$ 。设 $a_{j}(j=1 \cdots p)$ 表示 
指标体系的 $p$ 个指标, 共有 $n$ 个评估对象。指标的量 化值结果为 $\left(\mu_{j k}\right)_{p \times n}(j=1 \cdots p ; k=1 \cdots n), \mu_{j k}$ 指第 $k$ 个评估对象的第 $j$ 个指标量化结果。在此次研究中, 分析指标量化结果, 取所有评估对象的各指标值的 $(0,20,40,60,80,100)$ 百分位数, 组成各风险评估云 的上界和下界的指标值集合, 用上一节的约束随机赋 权法得到的权重矩阵, 生成评语集风险序列, 得到评 语集定量变量的云化初始值, 取其期望值作为对应风 险等级指标集的上界和下界, 并通过正向云发生器 ${ }^{[25]}$ 得到五个评语集的云。云参数计算通过下式得到:

$$
\left\{\begin{array}{l}
E x=(a+b) / 2 \\
E n=(b-a) / 6 \\
H e=k
\end{array}\right.
$$

其中, $a$ 为约束的下边界, $b$ 为约束的上边界, $k$ 是常 数, 通过试验确定取值;对于只有单边约束条件的定量 变量, 可根据测试数据的最大上限和下限确定缺省边 界参数或期望值, 然后参照上式计算云参数, 用半升 半降云来描述 ${ }^{[21]}$ 。

在此次研究中, 经过定量指标云化产生的评语集 [极好, 好, 中等, 差, 极差]对应的云模型分别是:

Cloud $_{\text {极好 }}(0.0200,0.0288,0.001 \mid x>E x) ，$

Cloud $_{\text {好 }}(0.3204,0.0492,0.0016)$,

Cloud $_{\text {中等 }}(0.5432,0.0250,0.0026)$,

Cloud 差 $_{\text {差 }}(0.6967,0.0262,0.0016)$,

Cloud $_{\text {极差 }}(0.9200,0.0167,0.001 \mid x<E x)$ 。

上述云模型中的三个数字为其三个特征值, 例如 Cloud $_{\text {中等 }}(0.5432,0.0250,0.0026)$ 是对应评语为 “中等” 的云模型, 其期望为 0.5432 , 熵为 0.0250 , 超熵为 0.0026 。

\section{1 .2 浮动云得到评语集}

浮动云用于生成论域给定的语言值中没有覆盖的 空白区域的虚拟语言值 ${ }^{[26]}$ 。设论域 $U$ 中的两朵相邻基 云 $A_{1}\left(E x_{1}, E n_{1}, H e_{1}\right)$ 和 $A_{2}\left(E x_{2}, E n_{2}, H e_{2}\right)$, 则位于两朵 基云之间任意位置 $\mathrm{u}$ 存在一浮动云的数字特征由下式 计算:

$$
\left\{\begin{array}{l}
E x=u \\
E n=\frac{E n_{1}\left(E x_{2}-E x\right)+E n_{2}\left(E x-E x_{1}\right)}{E x_{2}-E x_{1}} \\
H e=\frac{H e_{1}\left(E x_{2}-E x\right)+H e_{2}\left(E x-E x_{1}\right)}{E x_{2}-E x_{1}}
\end{array}\right.
$$

将定量变量的云化的结果和浮动云得到的结 果还原的云模型置于同一坐标系中, 得到指标值取 在不同区间的云标尺。在本研究中, 以相邻两朵评 语集的云模型生成浮动云对应的评语集云模型分 别是:

$$
\begin{aligned}
& \text { Cloud }_{\text {很好 }}(0.1702,0.0390,0.0013), \\
& \text { Cloud }_{\text {较好 }}(0.4318,0.0371,0.0021), \\
& \text { Cloud 较差 }(0.6199,0.0256,0.0021), \\
& \text { Cloud 很差 }(0.8084,0.0214,0.0013) \text { 。 }
\end{aligned}
$$

其含义与定量变量云化得到的评语集云模型一致, 例 如, Cloud 很差 $(0.8084,0.0214,0.0013)$ 指评语为 “很 差” 的云, 其对应的云模型的期望值为 0.8084 , 熵为 0.0214 ，超熵为 0.0013 .

\subsection{3 正向云发生器和云标尺生成}

正向云发生器是从定性到定量的映射 (图 1), 根 据云的数字特征产生云滴。变量云化结果得到的云参 数通过正向云发生器, 便得到对应评语云。

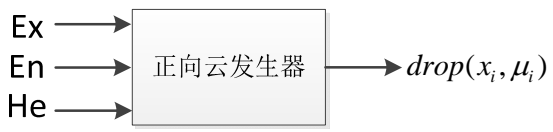

图 1 正向云发生器

Fig.1 Cloud generator

正向云发生器的具体步骤:

输入: 由 3.1.1 和 3.1.2 产生的风险序列对应的特 征值 $(E x, E n, H e)$; 输出: $n$ 个云滴 $x$ 及其确定度 $y$ 。

Step1:生成以 $E n$ 为期望值, $H e$ 为方差的正态随机 数 $E n n ; S t e p 2$ :生成以 $E x$ 为期望值, $E n n$ 为方差的正 态随机数 $x_{i}$; Step3: 计算 $y_{i}=e^{\frac{-\left(x_{i}-E x\right)^{2}}{2(E n n)^{2}}} ;$ Step4: $\left(x_{i}, y_{i}\right)$ 为一个云滴; Step5: 重复步骤 (1) (4), 直到产生 $\mathrm{n}$ 个要求的云滴。

本研究中用指标值云化以及浮动云通过正向云发 生器得到的云标尺(图 2), 图中的底部横坐标为风险大 小, 纵坐标为确定度。 


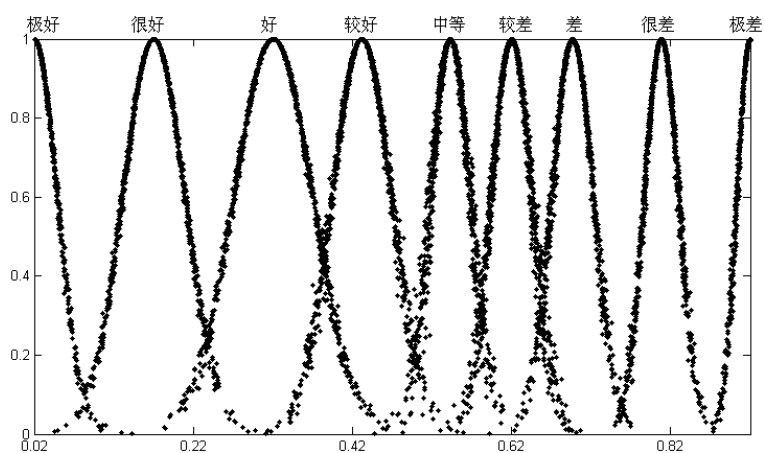

图 2 黄河流域水安全风险评语集云模型（云标尺）

Fig.2 Cloud model of the Huang River basin's water security risk level

\section{2 风险序列生成风险云}

\subsection{1 逆向云发生器}

逆向云发生器用于从已知的云滴中求出正向发生 器的三个特征值 $(E x, E n, H e)^{[18]}$, 实现定量值到定性概 念的转换 (图 3)。将风险序列作为无确定度信息的逆 向云发生器的输入, 得到该风险序列对应的风险云特 征值, 再用正向云发生器, 得到特征值相同的云作为 该风险序列的风险云。

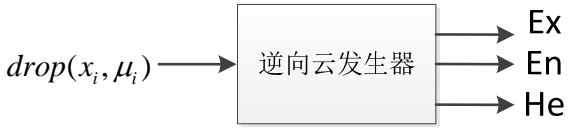

图 3 逆向云发生器

Fig.3 Reversed cloud model

设 $\left(R_{i k}\right)_{m \times n}$ 是所有评估对象的水安全风险矩阵, $R_{i k}$ 表示根据第 $\mathrm{i}$ 组指标权重值对第 $\mathrm{k}$ 个对象评估结果, 即 $\left(R_{i k}\right)_{m \times n}$ 的每一列代表对应的评估对象的风险序列。
以 $R_{k}$ 表示第 $k$ 个评估对象的风险序列, 作为第 $k$ 个评 估对象对逆向云发生器的输入项。具体做法如下 ${ }^{[27]}$ :

输入: 样本点 $x_{i}=R_{i k}$, 其中 $i=1,2, \cdots, n$ 。输出: 反应定性概念的数字特征 $(E x, E n, H e)$ 。

Step1:计算样本平均值 $\bar{X} \approx \frac{1}{n} \sum_{i=1}^{n} x_{i}$, 一阶样本绝对中 心距 $\frac{1}{n} \sum_{i=1}^{n}\left|x_{i}-\bar{X}\right|$, 样本方差 $S^{2}=\frac{1}{n-1} \sum_{i=1}^{n}\left(x_{i}-\bar{X}\right)^{2}$;

Step2: $E x=\bar{X}$; Setp3: $E n=\sqrt{\frac{\pi}{2}} \times \frac{1}{n} \sum_{i=1}^{n}\left|x_{i}-E x\right|$;

Step4: $H e=\sqrt{S^{2}-E n^{2}}$ 。

根据表 3 中各评估对象风险序列, 通过上述步骤 得到相应云模型数字特征值:

表 4 黄河流域八个省份风险云数字特征值

Table 4 Eigenvalues of the assessed provinces'risk clouds

\begin{tabular}{ccccccccc}
\hline 评估对象 & 山西 & 内蒙古 & 山东 & 河南 & 陕西 & 甘肃 & 青海 & 宁夏 \\
\hline $\mathrm{Ex}$ & 0.616 & 0.545 & 0.646 & 0.576 & 0.490 & 0.520 & 0.087 & 0.477 \\
$\mathrm{En}$ & 0.029 & 0.020 & 0.038 & 0.043 & 0.027 & 0.015 & 0.019 & 0.047 \\
$\mathrm{He}$ & 0.025 & 0.018 & 0.020 & 0.008 & 0.020 & 0.020 & 0.015 & 0.019 \\
\hline
\end{tabular}

\section{2 .2 风险云再生成}

再次通过 3.1 .3 中的正向云发生器, 得到特征值 与评估对象风险序列相同的风险云。将各评价对象的 风险云置于图 2 所示的云标尺中, 可得到特征值与风 险序列所含信息对应的风险云分布图 (图 4)。由此 产生特征值与风险序列所含信息对应风险云分布图。
由表 4 中的云模型数字特征, 通过正向云发生器 还原出个评估对象的云模型图像, 云的期望用云的整 体位置表示, 熵是图中云的离散程度, 超熵是云的厚 度, 其中, 横坐标为水安全风险的大小, 纵坐标为确 定程度: 


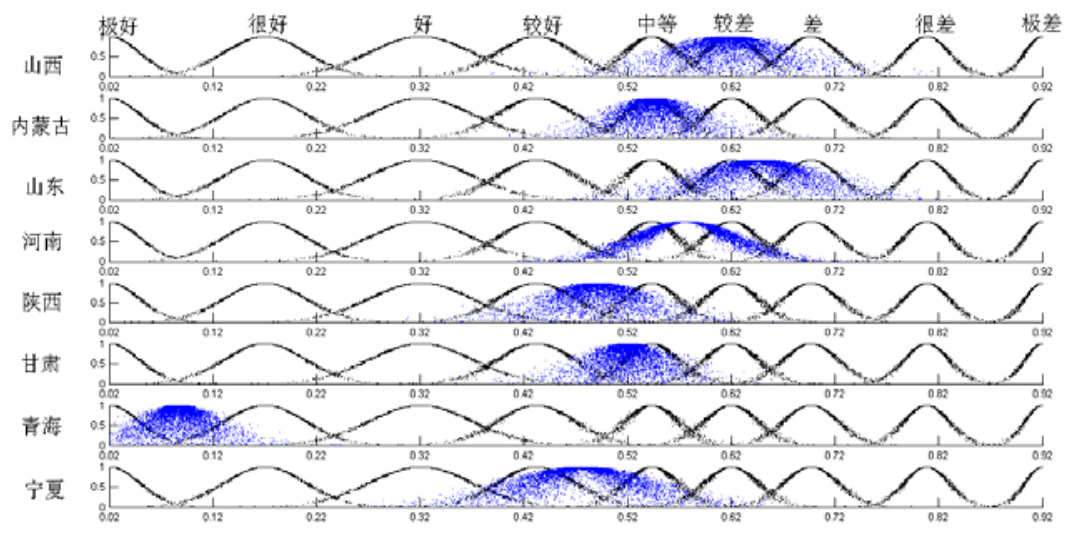

图 4 黄河流域八省水安全风险云模型

Fig.4 Cloud models of the eight provinces of the Huang River basin's water security risk

\section{3 结论与分析}

图 4 给出八个省份的风险云形状及风险云在评估 云标尺中所在位置。风险云的整体位置表示了风险的 大小, 云滴离散程度和厚度表示云滴对评语的隶属程 度和风险云所对应的语言概念的清晰程度。从风险云 和云标尺的对应关系来看, 山东水安全为八个省份中 最差, 其风险云分布于 “较差” 和 “差”之间; 山西 的水安全分布介于 “中等” 到 “差”之间, 其期望值 与 “较差” 等级十分贴近, 可将山西水安全风险等级 确定为 “较差”; 河南省位于 “较差” 和 “中等” 之间, 接近中等; 内蒙古风险云几乎覆盖了整个 “中等” 评 语集, 因此内蒙古的风险等级为 “中等”; 甘肃风险云 形状与内蒙接近, 中心位置较内蒙古稍偏左, 因此仍 将其风险等级定为“中等”, 事实上其风险小于内蒙古;
陕西和宁夏的风险云都位于 “中等” 和 “较好”之间, 偏向于 “较好”, 将此二者风险等级定位 “较好”, 但 需注意的是宁夏风险云覆盖范围比山西更广; 青海的 风险云位于 “极好” 和 “很好” 之间, 稍偏向于 “很 好”, 可将其风险等级确定为 “很好”。

图 5 为 ArcGis 软件下绘制出的风险区划图。黄河 发源于青藏高原, 青海省位于黄河源区, 水资源较为 丰沛, 同时人口稀少, 经济发展较下游地区相对缓慢, 社会需水量及水资源污染情况都相对较好, 水安全风 险的暴露性和脆弱性都很小, 因此水安全风险等级很 低; 山东省位于黄河流域的下游, 人口密集, 水资源 消耗大, 工业发展水平较高, 水资源的危险性和脆弱 性都很大; 山西省的水资源量本身较小, 而人口基数 大, 同时节水措施不够完善, 危险性和脆弱性都很大, 而防范能力较低, 导致山西省的水安全风险等级较高。

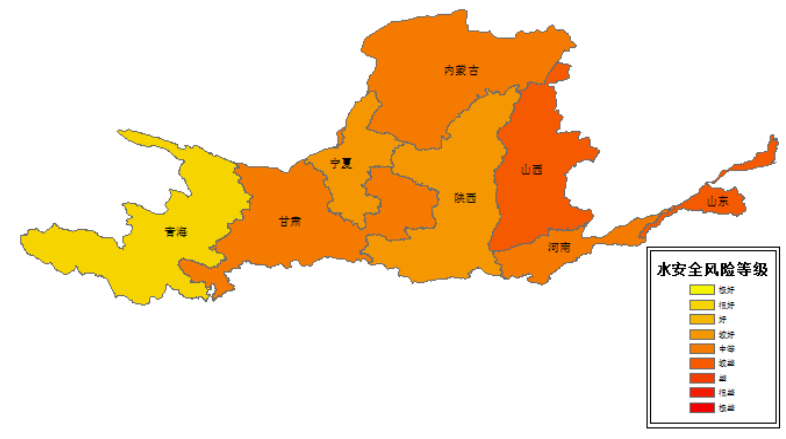

图5 黄河流域水安全风险区划

Fig 5 Water security risk zoning of the Huang River basin

\section{5 小结}

通过水安全的风险因子分析, 建立了风险评估指 标体系, 在此基础之上对指标值特性进行分析并结合
经验知识和专家建议, 确定了四个指标赋权的约束条 件, 在满足约束条件的基础上随机大量产生权重序列, 得到各评估对象的风险序列。

对指标权重进行分析, 通过定量指标云化和浮动 云生成算法, 产生了风险评语集合云模型。对风险序 
列通过逆向和正向云发生器得到与各指标风险序列特 征值相对应的八朵风险云。最终得到所有评估对象的 风险等级。

常规方法对水安全的评估一般从水安全的定义和 内涵出发, 构建指标体系, 利用层次分析法、模糊综 合评价等方法确定评价对象的水安全风险大小, 最终 得到的结果一般是将某区域的水安全定为 “非常安 全”、“基本安全”、“不安全” 等明确的评语等级。本 文尝试使用随机赋权和云模型结合的方法, 最终得到 的结果也包含了水安全风险评语等级的划分, 但在生 成风险云得到评估对象的风险等级的同时, 风险云模 型图中同时可得到风险等级以外的信息, 如风险评估 结果的离散性、评语的确定程度。约束随机赋权充分 利用现有知识体系的已知信息, 将风险量化成风险序 列并通过云模型将其云化成易于直观感受的风险云, 将风险的大小和不确定性融合并在定量-定性之间合 理转化, 在水安全风险评估表现出良好的适用性。

\section{参考文献}

1. Christina Cook,Karen Bakker.Water security:Debating an emerging paradigm.Global Environmental Change.2012(22):94-102.

2. Pang Yan-jun,Liu Kai-di,Zhang Bo-wen,The Metho of Determing the Objective Index Weight in the Synthetic Evaluation Sysstem[J]. Systems Engineering-Theory\& Practice.2001,8(8):37-42.

庞彦军, 刘开第, 张博文. 综合评估系统客观性指标权重 的确定方法[J].系统工程理论与实践.2001,8(8):37-42.

3. Wang Ze-yan,Gu Hong-fang,Yi Xiao-xin,et al.A Method of Determining the Linear Combination Weights Based on Entropy[J].Systems

Engineering-Theory\&Practice.2003,3:112-116. 汪泽炎, 顾红芳, 益晓新, 等.一种基于熵的线性组合赋 权法[J].系统工程理论与实践,2003,3:112-116.

4. MA Zhipeng,CHEN Shoulun, LI Xiaoying. Grey Decision Model of Flood Control Program Based on Random Weight.Water Resources and Power, 4(2007):41-44. 马志鹏, 陈守伦, 李晓英. 基于随机赋权法的洪水调度方 案灰色决策模型 $[\mathrm{J}]$. 水电能源科学,4(2007):41-44.

5. HAN Yuping,RUAN Benqing.A Preiminary Study on Index System of Regional Water Security Assessment. Acta Scientiae Circumstantiae,2(2003):267-272.

韩宇平, 阮本清. 区域水安全评估指标体系初步研究[J]. 环境科学学报,2(2003):267-272.

6. Li Lei.Intelligent Multi-Attribute Evaluation of Water Security[D].Hefei University of Technology.2007. 李磊.水安全智能多属性评价[D].合肥工业大学.2007.

7. TANG Jun, ZHAO Wenjie,LI Dejun.Comprehensive Effectiveness Evaluation of Warfare Intelligence Based on Cloud Model[J].Ship Electronic Engineering.6(2012):37-39,70.

汤君, 赵文杰, 李德军. 基于云模型的战场情报综合效能 评估[J].舰船电子工程. 6(2012):37-39,70.
8. WANG Shuyun. Study on Water Security Comprehensive Assessment[D].Nanjing:Nanjing Hydraulic Research Institute.2010.

王淑云.水安全综合平价研究[D].南京: 南京水利科学研 究院.2010.

9. 王远坤.水安全评价体系及其应用研究[D].青岛: 山东大 学.2006.

10. ZOU Hengwan.Risk Assessment of Climate Change Impacts on Water Resources and Water Disputes in China[D].Nanjing:PLA University of Science and Technology.2011.

邹恒婉.气候变化对中国水资源的影响与水资源争端风 险评估[D].南京: 解放军理工大学.2011.

11. JIA Shaofeng,ZHANG Junyan,ZHANG Shifeng.Regional Water Resources Stress and Water Resources Security Appraisement Indicators[J].Progress In Geography, 2002,21 (6) : 538-545.

贾绍风, 张军岩, 张士峰, 等. 区域水资源压力指数与水 资源安全评价指标体系[J]. 地理科学进展, 2002,21（6）: 538-545.

12. XIA Jun,ZHU Yizhong.The Measurement of Water Resources Security:A Study and Chanllenge on Water Resources Carrying Capacity[J].Journal of Natural Resources.2002,17(3),262-269.

夏军, 朱一中.水资源安全的度量: 水资源承载力的研究 与挑战 $[J]$.自然资源学报，2002,17（3），262-269.

13. ZENG Changyun,LEE Guibao.Study on Water Environment Security and Evaluation Index System_a Case Study of Beijing[D].Capital Normal University,2004. 曾畅云, 李贵宝.水环境安全及其指标体系研究——以北 京市为例[D].北京: 首都师范大学,2004.

14. ZHANG Qiaoxian,OUYANG Zhiyun,WANG Rusong,et,al.Modeling Water Security in China and Comparision of the Strategies[J].Advances In Water Science,2002,12(5):452-457.

张巧显, 欧阳志云, 王如松, 等.中国水安全系统模拟及 对策比较研究 [J].水科学进展, 2002,13（5）：569-577.

15. LIU Yidan,DONG Bidan,et,al. 刘一丹, 董碧丹, 等. 基于模糊评估的等级保护风险评估 模型[J].计算机工程与设计.2012,2（34）:452-457.

16. Yacov Y. Haimes.On the Comples Definition of Risk: A Systems-Based Approach.Risk Analysis.[J]2009,29 (12).

17. HU Haipeng.A New Method in Producing Pseudo Random Number and Their Statistical Performance[D]. Changsha:National University of Defense Technology,2007.

胡海朋. 一种新的伪随机数产生方法及其统计性能分析 [D].长沙: 国防科学技术大学.2007.

18. LIU Changyu,Li Deyi,Pan Lily.Uncertain Knowledge Representation Based on Cloud Model[J].Computer Engineering and Applications.2004,2. 刘常昱, 李德毅, 潘莉莉. 基于云模型的不确定性知识表 示 $[J]$.计算机工程与应用.2004, 2.

19. LI Deyi,Duyi.Artificial Intelligence with Uncertainty[M].National Defense Industry Press.2005,7. 李德毅, 杜貖鸟. 不确定性人工智能 [M]. 国防工业出版 社.2005, 7 . 
20. LI Deyi,SHI Xuemei,MENG Haijun.Membership Cloud and Membership Cloud Producer[J].Computer Research and Advance,1995,32(6):15-20.

李德毅, 史雪梅, 孟海军.隶属云和隶属云发生器 $[\mathrm{J}]$. 计 算机研究和发展,1995,32(6):15-20.

21. Li Deyi, Shi X M, Gupta M M. Soft Inference Mechanism Based on Cloud Models. In: Proceedings of the 1st International Workshop on Logic Programming and Soft Computing: Theory and Applications, Bonn, Germany, 1996.

22. Li Deyi, Han J, Shi X M. Knowledge Representation and Discovery Based on Linguistic Models. In:Lu H J, Motoda $\mathrm{H}$ eds.KDD:Techniques and Applications. Singapore: World Scientific Press, 1997, 3-20.

23. Li Deyi, Han Jiawei, Shi Xuemei, et al. Knowledge Representation and Discovery Based on Linguistic Atoms. Knowledge-based Systeme,1998(10): 431-440.

24. Li Deyi. Knowledge Representation in KDD Based on Linguistic Atoms. Journal of Computer Science and Technology,1997,12(6):481-496.

25. DU Xiangyu,YIN Quanjun,HUANG Kedi,LIANG Diannong.Transformation Between Qualitative Variables and Quantity Based on Cloud Models and its Application[J].Systes Engineering and Electronics,2008,30(4):772-776.

杜湘瑜, 尹全军, 黄柯棣, 梁甸农.基于云模型的定性定 量转换方法及其应用 [J]. 系统工程与电子技术 [J].2008,30(4):772-776.

26. Li Guofeng.Spatial Data Mining[D].Xi'an University of Electrical Science,2005.

李国锋. 空间数据挖掘技术研究 [D]. 西安电子科技大 学,2005.

27. LU Huijun,WANG Ye,LI Deyi,LIU Changyu.The Application of Backward Cloud in Qualitative Evaluation[J].Chinese Computers.2003,26(88):1009-1014.

吕辉军, 王晔, 李德毅, 刘常昱.逆向云在定性评价中的 应用[J].计算机学报. 2003,26(88):1009-1014. 